\title{
Sorting Out Sediment Grain Size and Plastic Pollution
}

\author{
BY HEATHER L. SPALDING, KANESA M. DUNCAN, AND ZOE NORCROSS-NU'U
}

\section{PURPOSE}

This activity familiarizes students with beach erosion, beach health, and the pervasive nature of plastic pollution. Students use measurements of sediment grain size and the buoyancy and density of materials found in beach sand to describe sediment size fraction, determine sand suitability for beach replenishment, and document the relative quantity of plastics. This activity also teaches field sampling (random sampling using transects and quadrats) and mathematical skills (graphing, percentages, and extrapolation).

\section{AUDIENCE}

The activity targets middle school and high school students but could be modified for undergraduate marine biology, oceanography, or geology courses. Moreover, although referencing ocean beaches, the principles and activities are relevant for both saltwater and freshwater coastlines. Students may use their own personal experiences with sand and beaches, the information included in this article, and information from the suggested Web sites to generate hypotheses about the effects of various coastal processes on sand formation and distribution.

\section{BACKGROUND}

Beach sand can appear fairly uniform, but it is actually a complex mixture of substances with various sizes and densities, and it comes from both abiotic (nonliving) and biotic (living) sources. Abiotic sources include igneous, sedimentary, and metamorphous rocks. Biotic sources include coral, algae, mollusks, and bryozoans (see Carter and Woodroffe, 1994). In fact, many white sand beaches, like those in Hawaii, are formed primarily from the carbonate skeletal remains of marine organisms. Examining the beach sand can tell us something about the local biology; in many tropical locations, the oatmealshaped flakes in the sand come from the green, calcified alga Halimeda. The presence of these sand flakes, or segments, means that Halimeda is growing nearby. Understanding sand composition, and the distribution of grain sizes, can also help in understanding the oceanographic processes shaping the coastline in a particular area. Higher-energy waves produce beach surfaces with a relatively homogenous, or similar, grain size distribution, whereas lower-energy waves produce surfaces with a more heterogeneous, or mixed, size distribution. At a particular beach, a greater proportion of finer sand grains may be pushed higher up the beach by waves while coarser grains are deposited closer to the water (Komar, 1977). A simple rule of thumb is that beaches exposed to high wave energy (longer wavelengths) tend to have coarser sediments than those exposed to lower wave energy. However, beaches are complex and highly variable environments, and there are conditions under which this rule doesn't hold true. The steepness of the beach may affect the intensity of the wave energy, and thus influence grain size distribution and sediment transport (Anfuso, 2005). Sediment availability is a critical factor in determining beach characteristics, and beach sediments often represent past conditions that are out of sync with current wave conditions. In Hawaii, much of the sand on beaches today was deposited by waves thousands of years ago (Harney et al., 2000). Additional factors influencing grain size include the nearshore and offshore bathymetry, bottom type, sand source, currents, wind exposure, and coastline shape.

Heather L. Spalding (hspaldin@hawaii.edu) is a graduate student in the Botany Department and a graduate teaching fellow in the NSF GK-12 Program, University of Hawai'i at Manoa, Honolulu, HI, USA. Kanesa M. Duncan is Assistant Professor of Science Education, Curriculum Research \& Development Group, University of Hawai'i at Manoa and Director, University of Hawai'i Sea Grant Center for Marine Science Education, Honolulu, HI, USA. Zoe Norcross-Nu'u is a coastal geologist and University of Hawai'i Sea Grant Extension Agent for Maui County, HI, USA. 
Sand on a beach may disappear, or erode, due to increased storm activity, high waves, human interruption of coastal processes, or sea-level rise (Figure 1). Knowing the grainsize distribution of the sand is important, not only for understanding a beach's ecology but also for knowing how best to replenish sand on a beach that is "disappearing." There are regulations for sand replenishment, or nourishment, in most areas. For example, the State of Hawaii requires that sand used for beach replenishment match the original grain size distribution within $20 \%$. Changing the characteristics of the sand too much may affect the habitat of organisms that live in the beach environment, such as burrowing sand crabs and turtles that dig holes in the sand to lay their eggs. It is also important that sand used for beach replenishment contain only

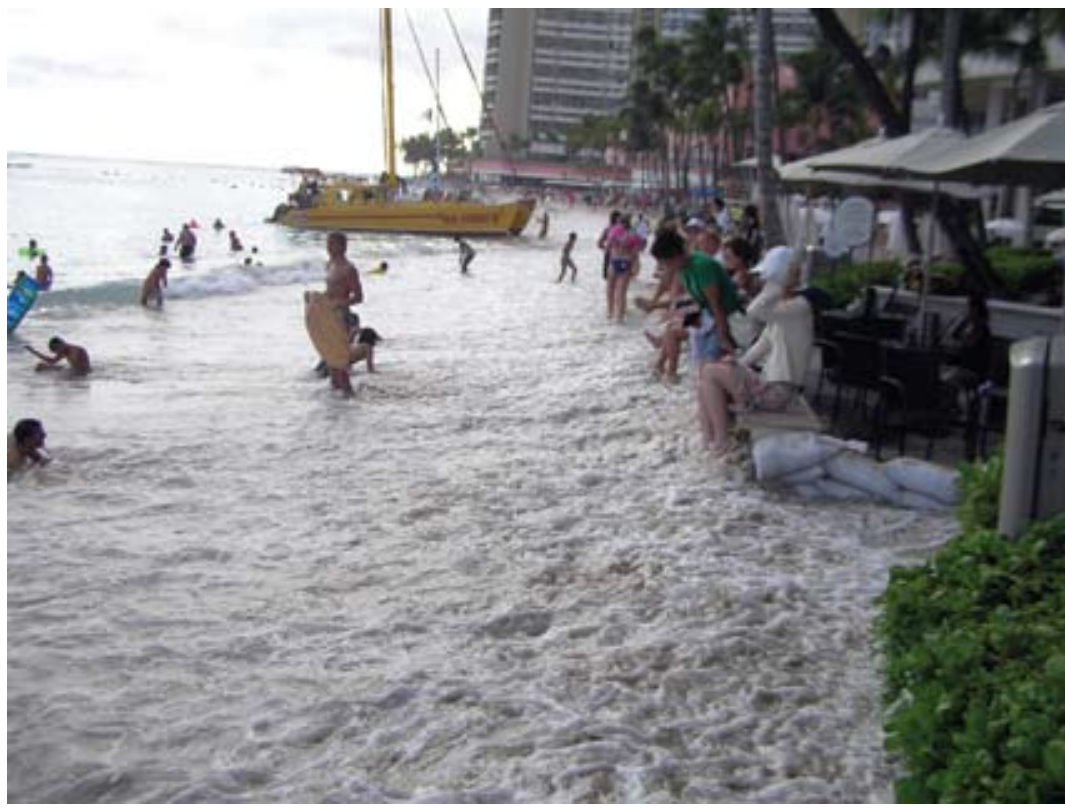

Figure 1. Beach loss caused by sand deficiency, high tide, and wave action, intensified by coastal development near to the water's edge, Waikiki, Hawai'i. Photo by C. Conger a small amount of fine particles because fine sediment can stay suspended in water for minutes, hours, or longer, depending on oceanographic conditions. Particles that are smaller than sand, such as silt and clay, can stay suspended for days to weeks, depending on conditions. The turbidity caused by these fine particles can be harmful to marine life by blocking out the sunlight necessary for photosynthesis-the process by which many corals and algae make the energy they need to grow. Fine particles settling on the ocean floor can also smother sensitive marine life. Generally speaking, sand with less than $6 \%$ fine particles (by mass) is considered acceptable for use in beach replenishment in Hawaii. At the other end of the spectrum, coarse gravel may settle quickly but be unappealing for recreational use.

Beach sand also contains many materials that are anthropogenic, or result from human influence, such as plastics. The quantity of plastics in beach sand may be influenced by proximity to streams and urban areas and the extent of human use, as well as ocean currents and wind directions. Plastics are dangerous in nature because they do not readily break down, or biodegrade. Marine life and seabirds can be harmed by consuming discarded plastics or by becoming entangled in them. In fact, a single piece of plastic may remain in the aquatic environment, polluting beaches, for hundreds of years even though it is no longer visible to the human eye. This is because plastics split up into ever-smaller particles, but they do not easily break down to their elemental components. Recently, scientists discovered that microscopic pieces of plastic can be found everywhere in the ocean, even inside plankton, the keystone of the marine food chain. In some areas, the mass of plastic can be as much as 2.5-6.0 times that of plankton (Moore et al., 2001, 2002). Plastics also trap dangerous pollutants, and ingesting plastic therefore means ingesting concentrated pollutants (Rios et al., 2007).

One way to separate plastics and other anthropogenic materials from beach sediment is by comparing the mass per unit volume, or density, of each material with that of water. Objects capable of floating in water must have densities less than water. Archimedes' Principle states that the weight of an object immersed in a fluid will be less than its "true" weight. The true weight is the downward force exerted by gravity on the object. Within a fluid, however, this downward force is partially opposed by a net upward force that results from the displacement of this fluid by the object. The difference between these two weights is known as buoyancy (see Chapter 3 in KarpBoss et al., 2009, for more discussion on buoyancy and related hands-on activities). Thus, plastics less dense and more buoyant than the surrounding water will float on top of the water and can be removed. These plastics are particularly dangerous 
because they can disperse great distances and remain in the water column for long periods of time, biodegrading into smaller and smaller pieces. Dense plastics that are less buoyant than the surrounding water will sink and have to be physically removed from the sediment. This activity uses buoyancy, density, and size to physically separate particles at a macroscopic level where students can visualize particle differences that parallel phenomena occurring on a microscopic level.

\section{RESEARCH QUESTIONS}

What is the percentage of fine-, medium-, and coarse-grained sand in samples collected from the beach? Is grain size related to observed wave energy or the history of wave energy at that beach? How does grain size affect settling time? How does the density and buoyancy of the materials found in sand compare to water? What was the total mass of plastic per square meter and number of plastic pieces per square meter?

\section{HYPOTHESES}

Beaches with higher wave energy tend to have larger sediment grain size, while beaches with lower wave energy tend to have smaller sediment grains. Finer-grained sediment will have a longer settling time than larger-grained sediment. Plastics and other marine debris with a density less than seawater will be buoyant in seawater and float.

\section{MATERIALS}

Field Sampling

- Transect line $(\sim 25 \mathrm{~m})$

- Random numbers along transect (one number per desired sample)

- PVC quadrat $\left(0.25,0.5\right.$, or $\left.1 \mathrm{~m}^{2}\right)$

- Small shovel

- Ruler

- 5-gallon bucket for sand samples

- 3 sand sieves

- Shallow container (larger than the sieves' circumference in order to sift the last sand portion directly into this container)

- Containers with lids for sifted sand samples (4 per quadrat sampled)

- Permanent labeling marker

\section{Laboratory Sampling}

- Digital scale

- Tall, clear graduated cylinder

(i.e., $1000 \mathrm{ml}$, filled with water)

- Beaker for weighing sand

- Stopwatch

- Graph paper

- Tablespoon

Note: Sieves can be purchased from a scientific supply company (plastic sieve sets with metal mesh are relatively inexpensive $[\sim \$ 60]$ yet effective), or sieves can be made by gluing mesh onto plastic containers (such as large margarine containers) with the bottoms cut out. Different mesh sizes can approximate the variety of mesh sizes used in a laboratory sieve shaker. With three sieves, you will end up with four samples. Try to find mesh sizes that approximate the desired range of very coarse, coarse, and medium grained sediments (Table 1).

Table 1. Size categories of sand grains and other particulate material. This scale for measuring grain size is called the "Wentworth Article Size Classification for Sand." It uses a geometric interval of $1 / 2$ to define the limits of each size fraction.

\begin{tabular}{|c|c|c|}
\hline Category & Type & Size $(\mathrm{mm})$ \\
\hline Boulders & Boulders & $250-100$ \\
\hline \multirow{3}{*}{ Gravel } & Cobbles & $65-250$ \\
\hline & Pebbles & $4-65$ \\
\hline & Granules & $2-4$ \\
\hline \multirow{5}{*}{ Sand } & Very coarse sand & $1-2$ \\
\hline & Coarse sand & $0.5-1$ \\
\hline & Medium sand & $0.25-0.5$ \\
\hline & Fine sand & $0.125-0.25$ \\
\hline & Very fine sand & $0.0625-0.125$ \\
\hline \multirow{6}{*}{ Mud } & Coarse silt & $0.031-0.625$ \\
\hline & Medium silt & $0.156-0.031$ \\
\hline & Fine silt & $0.0078-0.156$ \\
\hline & Very fine silt & $0.0039-0.0078$ \\
\hline & Clay & $<0.0039$ \\
\hline & Dust & $<0.0005$ \\
\hline
\end{tabular}




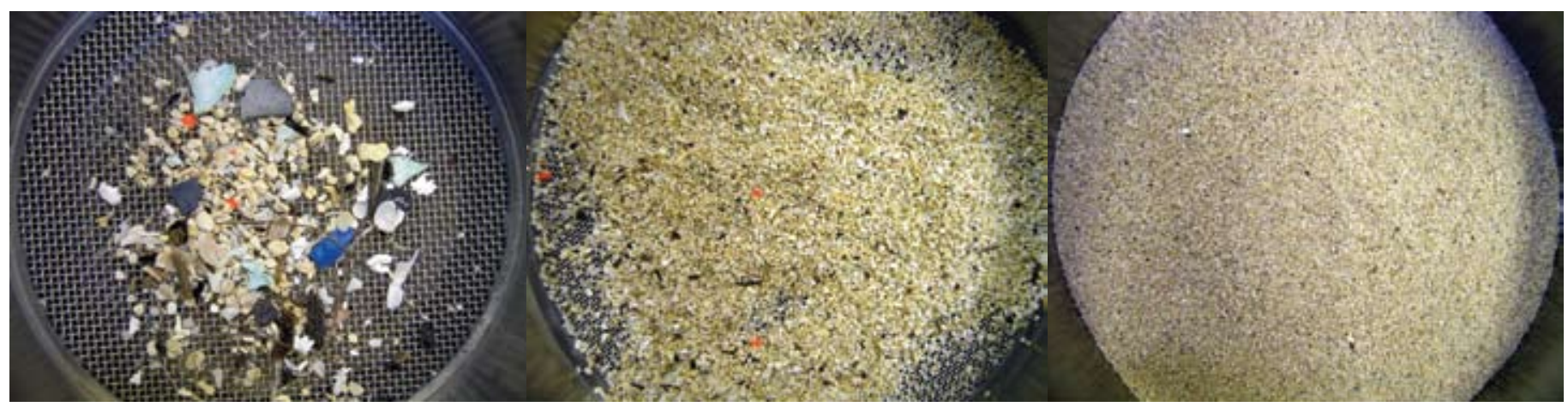

Figure 2. Beach sand sifted in sieves to show very coarse $(2 \mathrm{~mm})$, coarse $(1 \mathrm{~mm})$, and medium $(0.5 \mathrm{~mm})$ sand grains. Note the blue, black, light green, or orange plastic pieces in the very coarse and coarse sieves.

\section{ACTIVITY}

To initiate students into the process of scientific inquiry, the collection of sand samples should be as close to a real-world oceanographic setting as possible. They should discover the information provided in the background for themselves over the course of the activities-both through experimentation and authoritative inquiry (i.e., research and asking questions). Students can make observations and discuss hypotheses regarding grain size, beach sand stratification (larger grain size below smaller grain size), proportion of plastics in the sand, and wave energy based on their observations at the beach. They should become familiar with random sampling, transects, and quadrats. Students can discuss how the transect line should be placed on the beach (parallel or perpendicular to the water line) and determine the depth of sand sampled for each quadrat. We suggest a depth between $5 \mathrm{~cm}$ and $10 \mathrm{~cm}$, depending on the size of the quadrat.

\section{Sample Collection in the Field}

1. Students write down observations of beach wave energy; water turbidity; quantity of plastics; proximity to streams, rivers, or urban centers; and extent of human use. Students should also discuss how conditions at that beach might change throughout the year and what is known about the history of wave energy at that beach. Is it exposed to highenergy waves during part of the year, or is it relatively sheltered most of the time? These observations can be used in generating hypotheses about predicted sediment grain size. A $\mathrm{m}^{2}$ quadrat can be brought to the beach and placed on the sand to help students visualize the area covered by a square meter and the quantity of plastics in this area.
2. Lay the transect line along the beach in the orientation decided by the students.

3. Place the quadrat at the predetermined random number, and remove the sand with the small shovel to the desired depth. Place the sampled sand in the five-gallon bucket. Use the ruler to ensure the same depth is removed at each quadrat.

4. Use the sieves to sift the sand into the different grain sizes (Figure 2). If the sand is wet, water should be used to "wash" the samples through the sieves in order to separate the sand grains that are stuck together. Be sure to place the sieves in the correct order, from the largest (on top) to the smallest (on the bottom). Sift the smallest grain size into the shallow container. (Optional: Sand can be sifted in the classroom.)

5. Place the sifted sand sample from each sieve and the shallow container into separate containers. Label the top of each container with the grain size, quadrat number, and any relevant notes.

6. Repeat Steps 3-5 until the desired number of quadrats is sampled.

\section{Classroom Experiments}

Sediment Grain Size Distribution

1. Review students' hypotheses concerning grain-size distribution of the beach sand and the reasoning behind these hypotheses.

2. For each quadrat, weigh each sifted sand sample, record the weight, and return the sand samples to their containers (to be used for the following experiments).

3. Add the weight of sifted samples to find the total weight of sand for each quadrat.

4. Calculate the percentage total weight of each subsample. For 

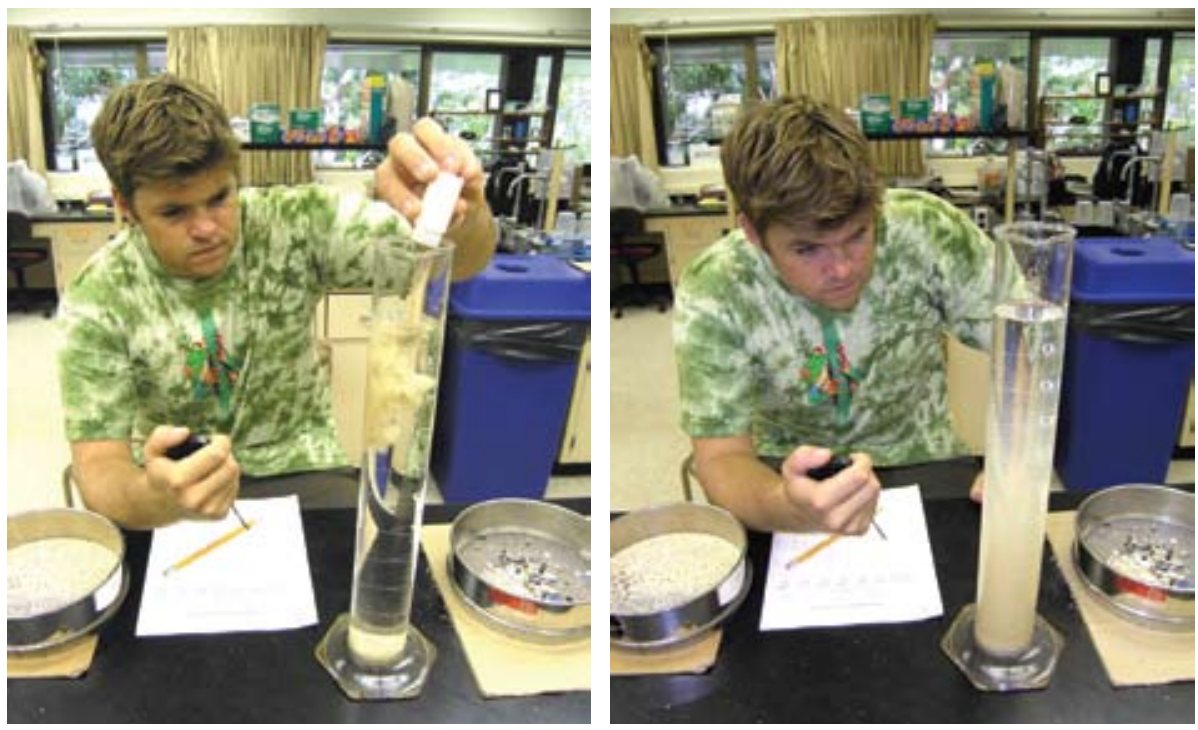

Figure 3. A student investigates the rate of sand particle settling by pouring 1 tablespoon of a sand sample into a graduated cylinder (left) and recording the amount of time it takes for the first and last particles of sand to reach the bottom of the cylinder (right).

example, if the total sand weight was $508 \mathrm{~g}$, and the coarse sand sample was $110 \mathrm{~g}$, then the percent coarse sand would be: $(110 \mathrm{~g} / 508 \mathrm{~g}) \times 100 \%=21 \%$.

5. Use percentage data to create a bar graph of sediment grain size distribution for each quadrat. Data can be averaged overall, or kept as separate quadrats, depending on the hypotheses developed by the students.

6. Have students evaluate their bar graphs, discuss whether their hypotheses were correct, and evaluate the processes (e.g., wave energy, recent storms, run-off) that might have influenced the sediment grain size distribution at the beach.

\section{Sand Particle Settling}

Now that students are familiar with the grain-size distribution of the sand, they can test the settling rate of different grain sizes. The settling rate represents the relative amount of time the sediment will remain suspended in the water column. Beaches with finer sediments tend to have more turbid water. (For more information on how the size of the particle may influence settling, see Boss et al., 2006.)

1. Have students formulate hypotheses concerning the differences in settling time for each sediment grain size.

2. Fill the large graduated cylinder with water.

3. Remove 1 tablespoon of sand from each sifted sample.

4. Starting with the largest sediment grain size, empty the tablespoon of sand into the water-filled graduated cylinder. 5. With a stopwatch, record the time it takes for the first and last sand particles to reach the bottom of the graduated cylinder. Repeat with each sifted sand sample. Record the settling times for each sample in a table. Rinse and refill the water-filled graduated cylinder for the plastic pollution experiment.

6. Have students evaluate their table, discuss whether their hypotheses were correct, and determine how the settling time of different sediment grain sizes may affect water clarity at a beach. If there was overlap between the slowest descent time of one sifted sample and the fastest descent time of the next smallest sifted sample, have the students discuss why this might have happened.

\section{Abundance, Density, and Buoyancy of Plastic Pollution}

Although it is not possible for middle school and high school students to directly observe the presence of microscopic plastic in the marine environment, they can replicate the observation of microscopic particles within plankton by discovering "hidden" macroscopic plastics in beach sediment. By placing sorted materials from beach sediment in water, students can determine whether the materials are more dense/less buoyant or less dense/more buoyant than water. They can compare their buoyancy results with the known density of these materials 
(Table 2), and extrapolate the amount of plastic pollution in a given area.

1. Review with students their observations and hypotheses regarding the amount of plastics on the beach per square meter.

2. Using the largest sediment grain size sample, sort and classify (if possible) each part of the sample (e.g., wood, different types of plastic, styrofoam, sand, shells, rope).

3. Count and weigh each particle that is anthropogenic in origin. Add up the total number of pieces and total weight of the anthropogenic materials. Extrapolate these values to $\mathrm{m}^{2}$ based upon the size of the quadrat used for sampling and the weight of the sediment grain size sample. Is this more or less than what was expected by the students?

4. For each type of particle, develop and record hypotheses regarding its buoyancy and density in water. For instance, if styrofoam is buoyant in the water, then it is less dense than water.

5. Test the hypotheses by placing sorted particles one by one into the water-filled graduated cylinder. Stir the water vigorously to break its surface tension. Lastly, place the sand into the graduated cylinder and note if any buoyant particles missed during the sorting are found. Record the results.

6. Have students evaluate their table and hypotheses, and compare their results with Table 1 . Were there any "hidden" particles found in the sand that were missed during the sorting? Discuss how the density and buoyancy of each anthropogenic object may have affected its transport to the beach or the distance traveled from its place of origin.

Table 2. Density of materials commonly found in association with beach sediment.

\begin{tabular}{|l|c|}
\hline Material & Density $\left(\rho=\mathrm{kg} \mathrm{m}^{-3}\right)$ \\
\hline Styrofoam & $30-120$ \\
\hline Cork & $220-260$ \\
\hline Wood & $110-1330$ \\
\hline Plastics & $850-1400$ \\
\hline Freshwater & 1000 \\
\hline Seawater & 1035 \\
\hline Lead & 11,340 \\
\hline
\end{tabular}

\section{GENERAL COMMENTS}

- The estimated time to complete this lesson is one to two hours for the sand collection at the beach, and two to three hours for the classroom experiments. The experiments could easily be broken up into three shorter classes. This lesson worked well with groups of three to four students.

- We suggest removing no more than a 5-cm depth into the sediments for a $1 \mathrm{~m}^{2}$ quadrat. Otherwise, there will be a high quantity of sediment to sift and transport.

- Depending on the wave energy, sediment at a beach may be stratified, with larger grains sizes below smaller ones. Students should be aware of this possible stratification and discuss how it may affect their sampling (i.e., the depth of sediment removed from the quadrat). Some of these variables in the sampling are likely to generate healthy discussion among the students and between them and the teacher.

- Encourage students to vigorously sift small portions of the sand and check the quantity of sand in each sieve after sifting each small portion. If there is a high quantity of a particular grain size at the beach, then that sieve will fill up quickly compared to the other sieves, making sifting difficult.

- If your sediment sample has a high volume of silt or clay particles, then the actual particles will be difficult to see, and it will take a long period of time (i.e., hours) for the particles to settle to the bottom and the water to clear. If this is the case, then you can specify a time limit condition, such as $>10$ minutes, for particle settling.

- Beaches are dynamic environments and do not necessarily reflect average oceanographic conditions on the day visited by the students. Using a beach that is monitored by video cameras may increase the number of observations the students can make before the fieldtrip. See the Web site section for links to possible beach video monitoring sites. Students could also collect historical oceanographic data for their site, such as recent storm activity and known wave exposure. Some of this information may be common knowledge to people closely associated with coastal areas, such as surfers, fishermen, or marine scientists.

\section{POSSIBLE MODIFICATIONS}

The experimental sand collection can be modified to address a broad range of questions concerning differences in sediment grain size, settling rate, and amount of marine debris between beaches with different wave energies or different levels of 
human impact. The sand can also be collected at a single beach but from different zones along the beach.

After students are familiar with the methods and concepts of this lesson, a "Crime Scene Investigation (CSI)" lesson can be used as follow-up to learn more about beach replenishment and to strengthen mathematical skills. The suggested scenario is that the beach where the students collected their sand for the original lesson has mysteriously eroded overnight. As CSI sand investigators, the students are tasked with determining which sand sample from a collection of vials (provided by the teacher) is appropriate for the beach replenishment. (The vials of sand can be from surrounding beaches, or known mixtures of different sediment grain sizes.) The sand used for replenishment must be similar to the original sand and follow beach replenishment guidelines. For example, in Hawaii, beach replenishment sand must be $<6 \%$ fine sand $(0.074 \mathrm{~mm})$ and $<10 \%$ coarse sand $(4.74 \mathrm{~mm})$, and over half must be $>0.125 \mathrm{~mm}$ in size and within $\pm 20 \%$ of the grain size of the original sand. Students will need to determine the sediment grain size distribution from the sand vials, and find the one best suited for beach replenishment. The lesson can involve a discussion about why these standards exist, what would happen if too coarse or too fine sand were used, and how differences in sediment grain size affect the ecology of the beach.

\section{RELATED TOPICS FOR FUTURE INVESTIGATIONS}

- Effect of particle shape and surface area on sediment settling speed and motion (see Boss et al., 2006)

- Impact of plastic debris on the world ocean, such as marine debris in the North Pacific Central Gyre (see Plastic Pollution and Marine Debris Web Sites below)

- Climate change impacts on coastal erosion and beach loss

\section{ADDITIONAL LITERATURE AND RESOURCES}

\section{Beach Video Monitoring Web Sites}

- http://www.planetargus.com

- http://www.earthcam.com

Individual surf and beach cameras can be found for your local area on the Internet, such as by searching for "Honolulu Web Cam" and finding Kuhio Beach, Waikiki, on the City and County of Honolulu Web site: http://www.honolulu. gov/cameras.
Plastic Pollution and Marine Debris Web Sites

- http://www.b-e-a-c-h.org/marine_debris.html

- http://marinedebris.noaa.gov

- http://www.unep.org/regionalseas/marinelitter/default.asp

Beach Sand Web Sites

- http://hawaii.gov/dlnr/occl/manuals-reports/sand-in-hawaii

- http://www.nytimes.com/2009/01/06/science/06prof. html?_r=3\&8dpc

- http://www.sandcollectors.org/ISCSHomeIndexx.html

Intertidal Transect and Quadrat Sampling

- http://www.hawaii.edu/gk-12/opihi/classroom_home.shtml

\section{ACKNOWLEDGEMENTS}

A modified version of this activity was presented at the “Teaching Science as Inquiry” teachers' workshop conducted in Maui, Hawaii, by the Maui Economic Development Board, the University of Hawaii at Manoa Curriculum Research \& Development Group, the NSF GK-12 Program, and Hawaii Sea Grant. Seventh-grade teacher Maggie Prevenas at Kalama Intermediate School in Maui, Hawaii, was the inspiration for this lesson. 迎

\section{REFERENCES}

Anfuso, G. 2005. Sediment-activation depth values for gentle and steep beaches. Marine Geology 220:101-112.

Boss, E., L. Karp-Boss, and P.A. Jumars. 2006. Settling of particles in aquatic environments: Low Reynolds numbers. Oceanography 19(2):151-154. Available online at: http://tos.org/hands-on/activities/19.2_boss_et_al.pdf (accessed August 27, 2009).

Carter, R.W.G., and C.D. Woodroffe, eds. 1994. Coastal Evolution. Cambridge University Press, Cambridge, 539 pp.

Harney, J.N., E.E. Grossman, B.M. Richmond, and C.H. Fletcher. 2000. Age and composition of carbonate shoreface sediments, Kailua Bay, Oahu, Hawaii. Coral Reefs 19:141-154.

Karp-Boss, L., E. Boss, H. Weller, J. Loftin, and J. Albright. 2009. Teaching Physical Concepts in Oceanography: An Inquiry-Based Approach. The Oceanography Society, Rockville, MD, 48 pp. Available online at: http://tos.org/hands-on/index. html (accessed August 27, 2009).

Komar, P.D. 1977. Selective longshore transport rates of different grain-size fractions within a beach. Journal of Sedimentary Research 47:1,444-1,453

Moore, C.J., S.L. Moore, M.K. Leecaster, and S.B. Weisberg. 2001. A comparison of plastic and plankton in the North Pacific Central Gyre. Marine Pollution Bulletin 42:1,297-1,300.

Moore, C.J., S.L. Moore, S.B. Weisberg, G.L. Lattin, and A.F. Zellers. 2002. A comparison of neustonic plastic and zooplankton abundance in southern California's coastal waters. Marine Pollution Bulletin 44:1,035-1,038.

Rios, L.M., C. Moore, and P.R. Jones. 2007. Persistent organic pollutants carried by synthetic polymers in the ocean environment. Marine Pollution Bulletin 54:1,230-1,237. 